\title{
International Federation for Emergency Medicine model curriculum for medical student education in emergency medicine
}

\author{
Cherri Hobgood • Venkataraman Anantharaman • \\ Glen Bandiera • Peter Cameron • Pinchas Halpern • \\ C. James Holliman • Nicholas Jouriles • Darren Kilroy • \\ Terrence Mulligan • Andrew Singer • \\ for the International Federation for Emergency \\ Medicine
}

Received: 24 September 2009 / Accepted: 4 November 2009 /Published online: 5 March 2010

(C) Springer-Verlag London Ltd 2010

\begin{abstract}
There is a critical and growing need for emergency physicians and emergency medicine resources worldwide. To meet this need, physicians must be trained to deliver time-sensitive interventions and lifesaving emergency care. Currently, there is no internationally recognized, standard curriculum that defines the basic minimum standards for emergency medicine education. To address this lack, the International Federation for Emergency Medicine (IFEM) convened
\end{abstract}

The views expressed in this paper are those of the author(s) and not those of the editors, editorial board or publisher.

\section{Hobgood $(\square)$}

Department of Emergency Medicine,

University of North Carolina School of Medicine,

CB 7594, UNC Hospitals,

Chapel Hill, NC 27599, USA

e-mail: hobgood@med.unc.edu

V. Anantharaman

Singapore General Hospital,

Singapore, Singapore

G. Bandiera

St. Michael's Hospital, University of Toronto,

Toronto, ON, Canada

\section{P. Cameron}

The Alfred Hospital Emergency and Trauma Centre,

Monash University,

Melbourne, Australia

P. Halpern

Tel Aviv Medical Center,

Tel Aviv, Israel a committee of international physicians, health professionals, and other experts in emergency medicine and international emergency medicine development to outline a curriculum for foundation training of medical students in emergency medicine. This curriculum document represents the consensus of recommendations by this committee. The curriculum is designed with a focus on the basic minimum emergency medicine educational content that any medical school should be delivering to

\section{J. Holliman}

Uniformed Services University of the Health Sciences,

Bethesda, MD, USA

N. Jouriles

Akron General Medical Center,

Akron, OH, USA

D. Kilroy

College of Emergency Medicine,

London, UK

T. Mulligan

Erasmus University School of Medicine,

Rotterdam, The Netherlands
A. Singer
Canberra Hospital,
Woden, Australia 
its students during their undergraduate years of training. It is not designed to be prescriptive, but to assist educators and emergency medicine leadership in advancing physician education in basic emergency medicine content. The content would be relevant, not just for communities with mature emergency medicine systems, but also for developing nations or for nations seeking to expand emergency medicine within current educational structures. We anticipate that there will be wide variability in how this curriculum is implemented and taught, reflecting the existing educational milieu, the resources available, and the goals of the institutions' educational leadership.

Keywords Curriculum · International emergency medicine . Medical education $\cdot$ Medical students

\section{Executive summary}

Vision: To create an international model curriculum for medical student foundation training in emergency medicine.

Rationale: There is critical, overwhelming, and growing need for emergency physicians and other administrative, professional, clinical, and academic emergency medicine resources worldwide. Currently, there exist a small number of national curricula for emergency medicine, but there is no standard, widely recognized international curriculum for medical students.

Demand: Currently worldwide, there are roughly $50+$ countries involved in the processes of emergency medicine development. Internationally, a consensus is building regarding the demand for an international minimum basic standard for emergency medicine curriculum content.

Goal: To establish, develop, and maintain an international curriculum for medical student foundation training in emergency medicine. The curriculum should be compiled by an international consortium of physicians, health professionals, and other experts in emergency medicine and international emergency medicine development. Further it should be approved, amended, and maintained by an international collection of such experts.

Endpoint: To further train and educate physicians, medical professionals, and other experts in emergency medicine in order to provide the best quality emergency care in the multiple and growing number of nations where it is currently practiced and to further establish emergency medicine as a medical profession worldwide.

\section{Mission statement}

The International Federation for Emergency Medicine believes that:

Society has a right to expect that at the completion of their undergraduate medical school training all physicians possess the basic knowledge of emergency care and the skills to manage common acute problems.

Emergency medicine is a core medical discipline and should be a required portion of the curriculum for every medical school, and every medical student, in the world.

Every physician, and graduating medical student, should be able to provide care in an emergency situation without any faults or lack of confidence and should be independent of the site of the emergency. Every physician, and medical student, should be able to manage clinical decision-making under pressure of time when it is essential to save lives.

Competence in basic emergency medicine should be an outcome measure for all medical students and represent a criterion required for conferral of the degree.

\section{Introduction}

This curriculum establishes an international consensus on the core content of undergraduate level emergency medicine training with the goal of elevating the quality of acute care worldwide through an expansion of basic emergency medicine education. This curriculum further reflects the importance of emergency medicine as a medical profession worldwide. The document is organized sequentially, as a framework rather than a comprehensive plan. Educators using this curriculum should make use of the framework to develop educational programs that are contextualized and specifically meet local educational requirements. This model allows easy adaptation of any of the features and provides an example of an expanded 4-year curriculum for a single learning objective.

\section{Professional development}

The clinical settings and environmental context for medical education varies widely throughout the world. To attain minimum basic competency in emergency medicine core learning objectives, medical students must be given a variety of opportunities for professional development. These opportunities should be longitudinal in nature, begin early in the preclinical years, and extend into clinical 
contexts that allow focus on acute and emergency conditions. The following basic guidelines should structure the educational process of achieving core competencies in minimum emergency medicine knowledge and skills.

During undergraduate and early training every medical student should:

- Acquire a fundamental knowledge of basic sciences as applied to emergency medicine and have the ability to assess and immediately treat common emergencies.

- Develop existing clinical examination skills and apply them in clinical practice to develop differential diagnoses and provisional management plans for acute medical conditions and undifferentiated patients.

- Acquire expertise in a range of commonly used emergency procedural skills, including basic life support.

- Perform allocated tasks, learn to process serially so as to optimally manage time within the shift, and meet clinical deadlines.

- Teach informally in the clinical setting and in specified circumstances in a more formal setting.

- Develop an understanding and basic awareness of clinical management issues when applied to acute care situations.

- Select and perform simple audit projects and understand the audit cycle to monitor care delivery and improve care quality.

- Understand the principles of critical appraisal and research methodology and apply these to acute care situations.

- Demonstrate the capacity to work in multiprofessional teams.

- Learn to recognize his or her own limitations in the provision of emergency care.

\section{Educational outcomes-learning objectives}

These learning objectives are designed to allow easy modification to the local needs and are written so that objective measures of performance and competency can be designed to measure attainment of the learning objective.

\section{The student should}

1. Acquire basic life support skills, including the diagnosis and treatment of shock and the related basic procedural skills, and demonstrate the basic application of these principles in real or simulated patient care scenarios.
2. Demonstrate the capacity to differentiate and treat common acute problems.

3. Provide a comprehensive assessment of the undifferentiated patient.

4. Demonstrate proficiency in basic life support skills and cardiopulmonary resuscitation.

5. Recognize and initiate first aid for airway obstruction.

6. Recognize and be prepared to intervene for all causes of shock in any age group.

7. Be able to provide rapid stabilization with intravenous access and fluid/blood administration.

8. Understand the principles of cerebral resuscitation in brain illness and injury.

9. Demonstrate proficiency in the use of an automatic external defibrillator (AED).

10. Understand the principles of wound care.

11. Demonstrate basic wound care techniques.

12. Understand the principles of trauma management.

13. Demonstrate basic trauma management skills, such as initial assessment using the $\mathrm{ABC}$ approach and full spine immobilization.

14. Demonstrate mastery of basic procedural skills, such as airway management and venous access.

15. Recognize life-threatening illness or injury and apply basic principles of stabilization to the early management of these entities.

16. Demonstrate the capacity to prioritize attention to those patients with more urgent conditions.

17. Describe the importance of the emergency department as a key link between the general population and the health care system.

18. Understand the role of the situations that are unique to emergency medicine: acute critical illness, intoxicated patients, media, out-of-hospital personnel, death notification for sudden unexpected death, disaster, language barriers, environmental illness/injury, injury prevention, assessment of complex and undifferentiated patients, and ability to synthesize multiple and often incomplete sources of information to develop a management plan.

\section{Unique content areas for emergency medicine in foundation training}

Undifferentiated patient presentation

Time-constrained decision-making

Environmental illness and injury

Prehospital care

Transition point between community and hospital

Focused history and exam

Prioritized differential diagnoses 


\section{Lead role areas for emergency medicine in foundation training}

\author{
Acute illness \\ Acute injury \\ Disaster management \\ Death notification \\ Injury prevention \\ Medical decision-making \\ Resource utilization \\ Toxicology
}

\section{Example curriculum format}

To assist educators in crafting a curriculum that fits local needs, we have provided an example of a 4-year plan for a single learning objective. Educators may use this as a guide to construct individual-, national-, and institution-specific models for content delivery. This method is not intended to be prescriptive, but to provide a simple model for tailoring content to the unique educational models that exist throughout the world.

Learning objective \# 5: recognize and initiate first aid for airway obstruction

Curriculum year 1 :

Readings - basic life support manuals, basic first aid manuals (e.g., American Heart Association Advanced Life Support Manual, Dallas, TX, USA or equivalent manuals from the local community)

Performance indicators:

1. Obtain basic cardiac life support (BCLS) certification

2. Demonstrate chin lift

3. Demonstrate bag-valve mask ventilation

4. Demonstrate the ability to clear an obstructed airway

Curriculum year 2

Readings - pathophysiology of respiratory failure

Curriculum year 3 and/or 4

Readings - introduction to anesthesia, introduction to airway management

Performance indicators:

1. Demonstrate endotracheal intubation

2. List indications for intubation

3. List contraindications for intubation

4. Describe medications used for rapid sequence intubation

5. Describe the physiology of artificial ventilation

Outcome measures
At the time of graduation, the student will demonstrate the ability to:

- Manage an obstructed airway

- Manage a basic airway, and

- Perform an endotracheal intubation

This will be assessed by simulation on a mannequin or using direct observation of student skills by trained faculty during clinical situations.

\section{Undergraduate emergency medicine curriculum content}

Skills curriculum

1. Clinical care skills

1.1. History and examination

1.2. Documentation

1.3. Decision-making

1.4. Time management

1.5. Safe prescribing

1.6. Continuity of care

1.7. Therapeutic interventions

2. Communication skills

2.1. With colleagues

2.2. With patients and caregivers

2.3. Breaking bad news

2.4. Working with a team

3. Maintaining good medical practice-lifelong learning

3.1. Audit and clinical outcomes

3.2. Critical appraisal

3.3. Information management

4. Professional behavior and probity - professional attributes

4.1. Career and professional development

5. Ethics and legal

5.1. Do not attempt resuscitation (DNAR) and advanced directives

5.2. The competent adult

5.3. Informed consent

6. Education-developing learning for others

6.1. Basic educational information delivery

6.2. Assessment and appraisal

6.3. Feedback 
7. Maintaining good clinical care-risk management

7.1. Medicolegal issues

7.2. Confidentiality

Specialty-specific curriculum

1. Generic objectives for resuscitation

1.1. Resuscitation-airway

1.2. Cardiac arrest/peri-arrest

1.3. Shock-all varieties

1.4. Coma

2. Anesthetics and pain relief - pain management

2.1. Local anesthetic techniques

2.2. Safe conscious sedation

3. Wound management

3.1. Basic wound débridement and closure

3.2. Identification and treatment of infected wounds

4. Generic objectives for trauma
4.1. Major trauma
4.2. Head injury
4.3. Chest trauma
4.4. Abdominal trauma
4.5. Spinal injury
4.6. Maxillofacial trauma
4.7. Burns
4.8. Orthopedic trauma

5. Generic objectives for musculoskeletal conditions

5.1. Upper limb disorders

5.2. Lower limb and pelvis disorders

5.3. Spine and spinal cord conditions

6. Vascular emergencies

6.1. Arterial limb threat

6.2. Venous - deep venous thrombosis (DVT)

7. Abdominal conditions

7.1. Undifferentiated abdominal pain

7.2. Hematemesis/melena

7.3. Anal pain and rectal bleeding

7.4. Diverticulitis

7.5. Abdominal aortic aneurysm

8. Urology

8.1. Acute urinary retention or bladder obstruction

8.2. Nephrolithiasis and colic
9. Sexually transmitted diseases

9.1. Identification and initial treatment for endemic diseases

10. Eye problems

10.1. Acute conjunctivitis - bacterial and viral

10.2. Acute vision loss

10.3. Acute eye trauma including globe rupture

11. Ear, nose, and throat (ENT) conditions

11.1. Epistaxis

11.2. Infections of the head and neck

12. Dental emergencies

12.1. Dental abscess

12.2. Dental fracture

13. Gynecology

13.1. Pelvic pain

13.2. Dysfunctional uterine bleeding

14. Obstetrics

14.1. Ectopic pregnancy

14.2. Uncomplicated emergency vaginal delivery

15. Cardiology

15.1. Basic electrocardiographic analysis

15.2. Recognition and initial treatment of acute myocardial infarction

15.3. Recognition and initial treatment of life-threatening arrhythmia

16. Respiratory medicine

16.1. Airway obstruction

16.2. Respiratory failure

16.3. Asthma and restrictive airway disease

16.4. Acute pneumothorax

16.5. Pulmonary embolism

17. Neurological emergencies

17.1. Acute stroke

17.2. Spinal cord lesions

17.3. Peripheral neuropathies

17.4. Acute mental status change

17.5. Migraine

17.6. Meningitis

17.7. Vertigo

18. Hepatic disorders

18.1. Acute hepatitis

18.2. Liver failure

18.3. Acute cholecystitis and cholangitis 
19. Toxicology

19.1. Treatment of acute ingestions

19.2. Identification of basic toxidromes

20. Acid-base and ventilatory disorders

20.1. Identification of acid-base disorders

20.2. Initial management of the mechanically ventilated patient

21. Fluid and electrolytes

21.1. Basic principles of fluid administration

21.2. Dehydration

21.3. Hyperkalemia

21.4. Hyponatremia

22. Renal disease

22.1. Acute renal failure

23. Diabetes and endocrinology

23.1. Disorders of glucose metabolism

23.2. Thyroid disorders

24. Hematology

24.1. Anemia

24.2. Disorders of red cell function

24.3. Disorders of clotting

25. Infectious diseases and sepsis

25.1. Endemic infectious diseases

25.2. Sepsis

25.3. Common infectious diseases or conditions (e.g., pneumonia)

25.4. Cellulitis and gangrene

26. Dermatology

26.1. Blistering and exfoliative diseases

26.2. Differential diagnosis of rash

26.3. Parasitic conditions and infestations

27. Rheumatology and immunology

27.1. Crystal arthropathy

27.2. Arthritis

27.3. Immune disorders

27.4. Anaphylaxis

28. Child protection and children in special circumstances

28.1. Child abuse signs and symptoms

28.2. Legal rights of parents to refuse care

29. Neonatology

29.1. Neonatal resuscitation

29.2. Hyperbilirubinemia
29.3. Disorders of feeding

29.4. Neonatal fever

30. Environmental emergencies

30.1. Hyperthermia

30.2. Hypothermia and frostbite

30.3. Envenomation and environmental toxin exposure

31. Oncology

31.1. Acute leukemia

31.2. Neutropenia and neutropenic fever

31.3. Solid tumors

31.4. Complications of chemotherapeutic agents

32. Pediatrics

32.1. Basic management of pediatric airway

32.2. Basic pediatric resuscitation

32.3. Common infectious diseases of childhood

32.4. Fever in the first 6 months of life

32.5. Common injury patterns for normal children

33. Psychiatry

33.1. Acute psychosis

33.2. Mood disorders

33.3. Personality disorders

33.4. Acute suicidal and homicidal ideation

33.5. Substance abuse

34. Major incident management

34.1. Concepts and application of triage

34.2. Field to hospital communication and chain of command

35. Legal aspects of emergency medicine

35.1. Refusal of care

35.2. Informed consent

35.3. Malpractice

36. Research

36.1. Formulating a research question

36.2. Review of the medical literature

36.3. Basic research design

36.4. Basic preparation of manuscripts and written publications

37. Management

37.1. Leading teams and giving orders

37.2. Basic concepts of debriefing and giving feedback

37.3. Time flow management 


\section{Selected references}

1. The Foundation Programme Committee of the Academy of Medical Royal Colleges. Curriculum for the Foundation Years in Post Graduate Education and Training. Academy of Medical Royal Colleges. Available via http://www.dh.gov.uk/prod_consum_dh/ groups/dh_digitalassets/@dh/@en/documents/digitalasset/ dh_4107696.pdf. Accessed 22 Feb 2009

2. Liaison Committee on Medical Education. LCME Accreditation Standards (with annotations). Liaison Committee on Medical Education June 2008. Available via http://www.lcme.org/functionslist.htm. Accessed 22 Feb 2009
3. Frank JR. The CanMEDS 2005 Physician Competency Framework. Better Standard, Better Physicians, Better Care. The Royal College of Physicians and Surgeons of Canada. Accessed 1 Mar 2009

4. Manthey DE, Coates WC, Ander DS et al (2006) Report of the Task Force on National Fourth Year Medical Student Emergency Medicine Curriculum Guide. Ann Emerg Med 47(3):e1-e7

5. Hockberger RS, Binder LS, Chisholm CD et al (2005) The model of the clinical practice of emergency medicine: a 2-year update. Ann Emerg Med 45(6):659-674

6. Chapman DM, Hayden S, Sanders AB et al (2004) Integrating the Accreditation Council for Graduate Medical Education Core competencies into the model of the clinical practice of emergency medicine. Ann Emerg Med 43(6):756-769 